\title{
All-optical Multilevel Regeneration in Nonlinear Optical Loop Mirror
}

\author{
Feng Wen ${ }^{1,2,}$, Christos P. Tsekrekos ${ }^{1}$, Xingyu Zhou ${ }^{2}$, Baojian $W^{2}$, Mingyue Zhu ${ }^{2}$, Jing Zhang ${ }^{2}$, \\ Kun Qiu $^{2}$, Sergei K. Turitsyn ${ }^{1}$ and Stylianos Sygletos ${ }^{1}$
}

${ }^{1}$ Aston Institute of Photonic Technologies, Aston University, B4 7ET Birmingham, UK,

${ }^{2}$ Key Lab of Optical Fiber Sensing and Communication Networks, Ministry of Education, University of Electronic Science and Technology of China, 611731 Chengdu, China

*f.wen@aston.ac.uk

\begin{abstract}
An all-optical multilevel amplitude regenerator is optimized based on the transmission response of a nonlinear optical loop mirror (NOLM). Three regenerative regions defined by the power-transferfunction (PTF) slope of less than 1 are obtained, and noise suppression is shown for a PAM4 signal. Using amplitude dithering we were able to experimentally characterize the transfer function slope and confirm the theoretical predictions.
\end{abstract}

Keywords: All-optical regeneration, PAM4, Nonlinear optical loop mirror

\section{INTRODUCTION}

Advanced modulated signals of high spectral efficiency have been intensively studied over the recent years due to their potential for improving the transmission capacity of future fiber links [1]. Although they can push the single channel data rate towards $400 \mathrm{~Gb} / \mathrm{s}$ or $1 \mathrm{~Tb} / \mathrm{s}$, they are highly susceptible to degradations coming from amplified spontaneous emission (ASE) noise and Kerr nonlinearity. Digital signal processing based transceivers can only partially address this issue by compensating degradations of deterministic type [2]. On the other hand, all-optical regenerators are capable of removing both deterministic and random distortions and thus become a low-cost and power-saving solution for future high capacity optical networks. Current research has been focused on regeneration schemes for multilevel signals that are encoded in amplitude and/or phase. Although phasesensitive amplifiers (PSAs), with a staircase like transfer function, have experimentally allowed a 4-level phase regeneration [3-5], amplitude regeneration has been shown only for 2-level signals, based on the use of a nonlinear optical loop mirror (NOLM) [6].

NOLMs are well known for their capability to suppress amplitude noise [6-8] and to improve the extinction ratio of optical signals [9]. Furthermore, due to their phase-preserving characteristics they can be used to suppress amplitude distortions on phase encoded signals without any amplitude-to-phase noise transfer [6].
Therefore, NOLMs are promising elements for the development of schemes dealing with advanced modulated signals and they should be thoughtfully investigated. In this paper, the regeneration of pulse amplitude modulated four (PAM4) signals is discussed. Using a theoretical model we investigate the optimum parameters for achieving multilevel regenerative response from a single NOLM unit. Subsequently, we present experimentally the scheme and with the use of an amplitude dither method we monitor the slope of the corresponding power transfer function (PTF) and show the existence of three regenerative regions.

\section{OPERATION PRINCIPLE}

The experimental setup for a NOLM-based all-optical multilevel regenerator is demonstrated in Fig. 1. Based on this setup a theoretical model has been also derived for system design purposes [10]. It included an optical coupler (OC), a polarization controller (PC), a variable optical attenuator (VOA) and a 606m-length highly nonlinear fiber (HNLF). The ability to launch high signal powers into the NOLM is crucial for achieving multiple regenerative levels. However, during the transmission of the signal into the HNLF, stimulated Brillouin scattering (SBS) effects convert most of the continuous-wave (CW) pump into backward-travelling Stokes light preventing in a real experiment the Kerr-based nonlinear response [10]. The use of specially designed fibers, e.g. strained

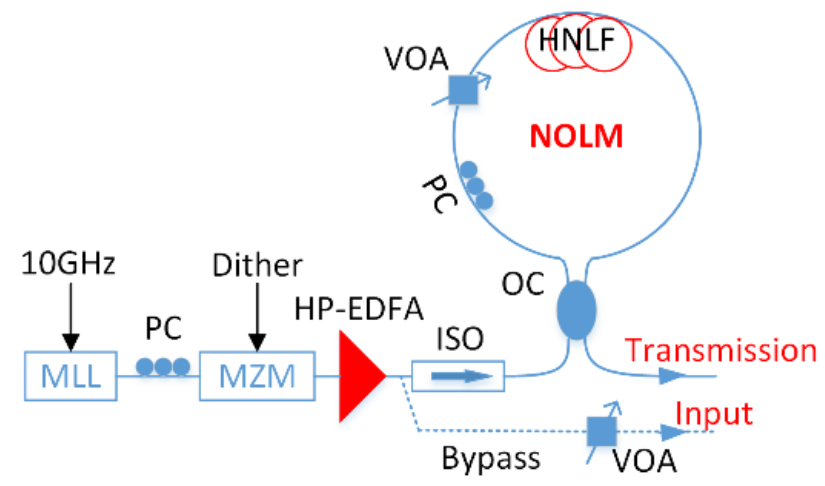

Fig. 1 Multilevel amplitude regenerator based on a NOLM unit. 
aluminous-silicate HNLF, along with pulsed signal operation helped to avoid the impact of the SBS effect. The nonlinear coefficient of the HNLF was $7 \mathrm{~W}^{-1} / \mathrm{km}$ and total fiber loss was $12 \mathrm{~dB}$. In the real experiment, the nonlinear response of the NOLM was not only determined by the Kerr-effect, but also by the birefringence of the fiber. Therefore, optimizing the polarization state of the input signal was critical for a smooth power transfer function curve with multiple plateau regions. In practice, this was achieved by launching a low-power signal, i.e. without inducing any nonlinear phase shift, at the input of the interferometer, and then tune the polarization controller to maximize the reflected power.

\section{THEORETICAL ANALYSIS}

The dependence of PTF on input signal power for different splitting ratios of the optical coupler (OC) of the NOLM is depicted in Fig. 2. This graph allows us to identify the operational peak power for each amplitude regenerative level. It can be noticed that : i) multiple power plateau regions are achieved for high input powers; ii) the power asymmetry between two arms of this NOLM helps to significantly reduce the required power; iii) a smoother PTF curve is obtained for larger power splitting ratio values. For the subsequent simulations of this paper, a PTF curve corresponding to a 0.95 splitting ratio was used to demonstrate the multilevel amplitude regeneration.

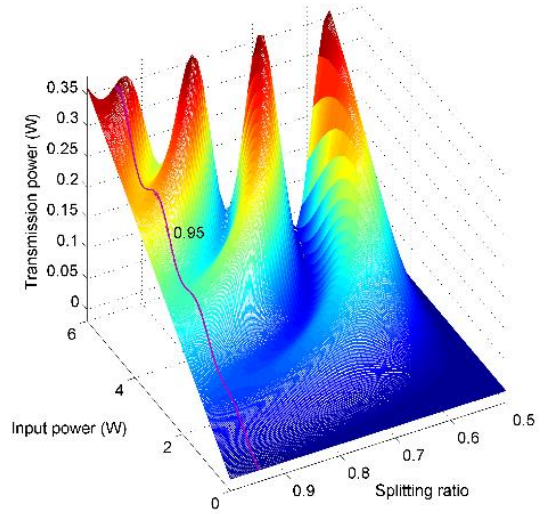

Fig. 2 Dependence of PTF results on input powers and splitting ratios.

To identify every plateau region, the slope was calculated on the normalized PTF curve, representing the amplitude noise power transfer ratio during the regeneration process. The PTF curve and its corresponding slope are depicted in Fig. 3. The regenerative region is defined by slope values less than one. Three power plateau regions $\mathrm{A}, \mathrm{B}$ and $\mathrm{C}$ were obtained matching the $2^{\text {nd }}, 3^{\text {rd }}$ and $4^{\text {th }}$ amplitude levels of a PAM4 signal, whereas no amplitude suppression was provided for the $1^{\text {st }}$ (i.e. zero) amplitude level of the PAM4. This corresponded to signal peak powers of 1.32, 2.94 and $4.58 \mathrm{~W}$ respectively, coinciding at the centers of the plateau regions. Moreover, the noise suppression capability of each of the three regenerative regions was different. The best noise suppression can be achieved at region $\mathrm{B}$ due to the smaller slope values.

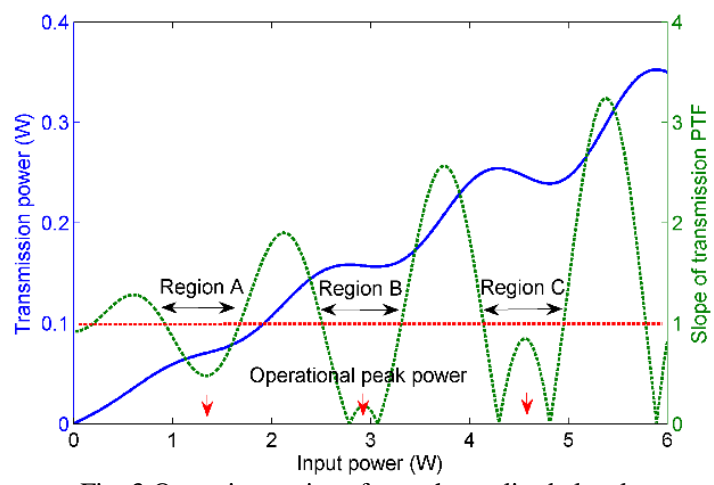

Fig. 3 Operation regions for each amplitude level.

Subsequently, we investigated the regenerative impact of an optimized NOLM transfer function, as shown in Fig. 3, on a RZ-PAM4 with equal distortion introduced at levels 2 to 4 . The eye diagrams of input and regenerated RZ-PAM4 signals are shown in Fig. 4. The red lines correspond to clean (i.e. undistorted) input pulses. Amplitude noise suppression is shown to be successfully achieved for this format. Table 1 depicts the normalized peak-to-peak power variation of each amplitude level before and after the regeneration. The noise power has been suppressed by $31 \%, 75 \%$ and $65 \%$, at the respective $2^{\text {nd }}, 3^{\text {rd }}$ and $4^{\text {th }}$ levels of the signal. Maximum suppression is achieved on the $3^{\text {rd }}$ signal level as predicted by the slope calculation on the PTF curve. Representing the change in the amplitude distance between symbols, the 'eye linearity', defined by the ratio of the largest to smallest mean voltage swing between symbols[11] was changed only by $5 \%$ compared to that of input signal. This indicates the potential for cascaded operation with minimum impact on the format shape.
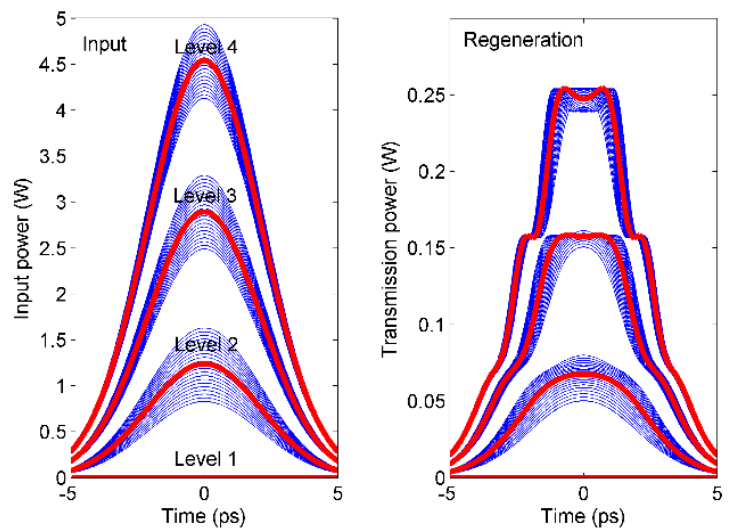

Fig. 4 Eye diagrams of input and regenerated signals.

Table 1 Normalized amplitude noises for each amplitude level

\begin{tabular}{|c|c|c|}
\hline $\begin{array}{c}\text { PAM4 amplitude } \\
\text { levels }\end{array}$ & Input & Regeneration \\
\hline Level 2 & 0.2775 & 0.1904 \\
\hline Level 3 & 0.2775 & 0.068 \\
\hline Level 4 & 0.2775 & 0.0981 \\
\hline
\end{tabular}




\section{EXPERIMENT}

The experimental characterization of the PTF slope was achieved by introducing amplitude distortion, through an optical modulator, on an input clock signal and measuring the corresponding suppression at the output, see Fig. 1. The radio frequency (RF) signal driving the amplitude modulator should be of small amplitude to avoid inducing large envelope variation on the optical clock which may influence the resolution of the slope measurement. In the experiment, a $10 \mathrm{GHz}$ optical clock of $10 \mathrm{ps}$ pulse width emitted by a fiber mode-locked laser (MLL) at 1550nm, was used. A $1 \mathrm{MHz}$ sine signal of $-12 \mathrm{dBm}$ modulated the optical clock stream. The optical power of the signal was controlled by a high-power Erbium-doped fiber amplifier (HP-EDFA). A 90:10 OC was used in the NOLM unit due to the smoother PTF obtained in a pulsed pump. This modulated optical clock was either input into the NOLM unit or bypass it for comparison. A VOA in the detour induced the same loss as the NOLM. The signal was detected by a $32 \mathrm{GHz}$ optical photodiode, and the RF power at $1 \mathrm{MHz}$ offset from the carrier was measured by a spectrum analyzer. We measured the power difference of the RF modulation at the input and output of the regenerator considering the fixed optical power. Fig. 5 depicts the results of this characterization. We can see the existence of three regenerative regions $\mathrm{A}, \mathrm{B}$ and $\mathrm{C}$ on the NOLM transfer function, which is in accordance with our theoretical predictions, potentially supporting the regeneration of PAM4 signals. Comparing the power difference values among three regions, the best amplitude noise suppression could be shown also for region B confirming the theoretical result.

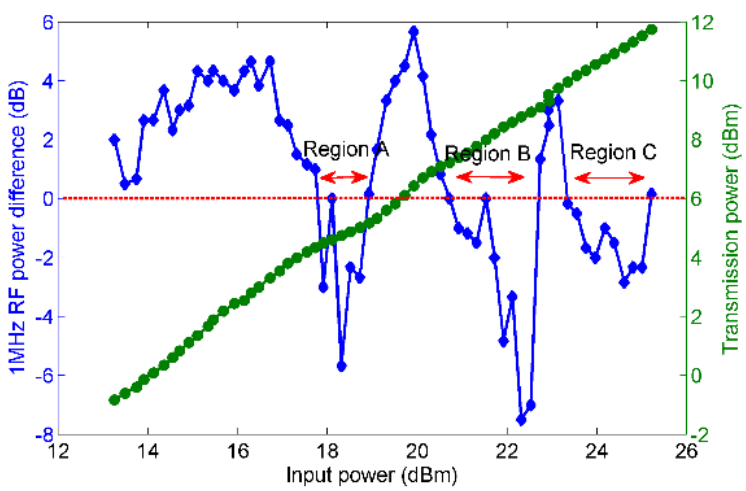

Fig. 5 Slope measurement using the dithering method and its PTF curve.

\section{CONCLUSION}

Multilevel amplitude regeneration for PAM4 signals was theoretically analyzed in an optimized NOLM unit. Amplitude noise suppressions at $2^{\text {nd }}, 3^{\text {rd }}$ and $4^{\text {th }}$ levels of the signal were simultaneously achieved. Guided by theory we implemented an optimized design of the NOLM and through a dithering method we characterized the regenerative properties of its transfer function. Three regenerative regions were also experimentally observed confirming the predictions of our theoretical model.

\section{ACKNOWLEDGMENTS}

The authors would like to thank the Tyndall National Institute for the loan of the highly nonlinear fiber, and $\mathrm{M}$. Tan, M. Sorokina, I. Phillips, F. Ferreira for valuable discussions. This work has been supported by the EPSRC project UNLOC (EP/J017582/1), the Marie SklodowskaCurie Action (701770-INNOVATION), the National Natural Science Foundation of China (61505021, 61671108), and the Program for Changjiang Scholars and Innovative Research Team in Universities of China (IRT1218), 111 Project (B14039).

\section{REFERENCES}

[1] P. J. Winzer, "Beyond 100G Ethernet," IEEE Commun. Mag., vol. 48, no. 7, pp. 26-30, 2010.

[2] E. M. Ip and J. M. Kahn, "Fiber Impairment Compensation Using Coherent Detection and Digital Signal Processing," J. Lightw. Technol., vol. 28, no. 4, pp. 502-519, 2010.

[3] M. Sorokina, S. Sygletos, A. D. Ellis, et al., "Optimal Packing for Cascaded Regenerative Transmission based on Phase Sensitive Amplifiers," Opt. Express, vol. 21, no. 25, pp. 31201-31211, 2013.

[4] A. Perentos, S. Fabbri, M. Sorokina, et al., "QPSK 3R Regenerator Using a Phase Sensitive Amplifier," Opt. Express, vol. 24, no. 15, pp. 16649-16658, 2016.

[5] F. Parmigiani, G. Hesketh, R. Slav' 1k, et al., "PolarizationAssisted Phase-Sensitive Processor," J. Lightw. Technol., vol. 33, no. 6, pp. 1166-1174, 2015.

[6] T. Roethlingshoefer, T. Richter, C. Schubert, et al., "Alloptical Phase-preserving Multilevel Amplitude Regeneration," Opt. Express, vol. 22, no. 22, pp. 27077 27085, 2014.

[7] M. D. Pelusi, Y. Matsui, and A. Suzuki, "Pedestal Suppression from Compressed Femtosecond Pulses Using a Nonlinear Fiber Loop Mirror," IEEE J. Quantum Electron., vol. 35, no. 6, pp. 867-874, 1999.

[8] A. Bogoni, P. Ghelfi, M. Scaffardi, et al., "All-Optical Regeneration and Demultiplexing for $160-\mathrm{Gb} / \mathrm{s}$ Transmission Systems Using a NOLM-Based Three-Stage Scheme," IEEE J. Sel. Top. Quantum Electron., vol. 10, no. 1, pp. 192-196, 2004.

[9] B. Olsson and P. A. Andrekson, "Extinction Ratio Improvement Using the Nonlinear Optical Loop Mirror," IEEE Photon. Technol. Lett., vol. 7, no. 1, pp. 120-122, 1995.

[10] F. Wen, S. Sygletos, M. Sorokina et al., "Multilevel Power Transfer Function Characterization of Nonlinear Optical Loop Mirror," Accepted by ICTON 2017, Girona, 2017.

[11] Tektronix, "PAM4 Signaling in High Speed Serial Technology: Test, Analysis, and Debug," Application note.http://tmsinc.co.kr/media/uploads/tek/application/[dp o70000sx]pam4_signaling_in_high_speed_serial_technol ogy_test_analysis_and_debug.pdf 